\title{
On an Algorithm for Solving Nonlinear Boundary- value Problem of Structural Analysis
}

\author{
Murtazaliyev Gelani Murtazaliyevich \\ Grand $\mathrm{PhD}$ in Engineering sciences, Professor \\ Dagestan State Technical University \\ Makhachkala, Russia \\ murtazaliev.gelani@mail.ru
}

Payzulayev Magomed Murtazaliyevich

$\mathrm{PhD}$ in Engineering sciences, Associate Professor

Dagestan State Technical University

Makhachkala, Russia

smdstu@mail.ru

\author{
Dibirgadzhiyev Anvar Magomedovich \\ Assistant lecturer \\ Dagestan State Technical University \\ Makhachkala, Russia \\ d.a.m.-001@mail.ru
}

\begin{abstract}
The emergence of modern high-strength materials leads to the creation of thin-walled structures in various fields of technology. To obtain the necessary information about their behavior under load, it is necessary to analyze all the characteristic features encountered at all stages of their loading at the initial (initial) stage of their work, considering one or several types of non-linearities, to find possible critical states, and to study the nature of the initial stage of postcritical deformation. The solution of such a general problem associated with discontinuous phenomena is based on mathematical ideas formulated in the branching theory of solutions of nonlinear equations.
\end{abstract}

Keywords - nonlinear solutions, sustainability, branching decisions, catastrophe theory, post-critic behavior.

\section{INTRODUCTION}

The fundamental mathematical concepts of the branching theory for solutions of nonlinear problems are considered in detail in the three main parts of this theory, indicated in Fig. 1.

In accordance with this scheme, the solution of the general nonlinear boundary-value problem of structural analysis associated with discontinuous phenomena is carried out because of the algorithm indicated in Fig. 2.

When calculating thin-walled structures, the study of the stability of equilibrium forms acquires special significance. In view of the variety of tasks being solved, the following classification of problem statements is possible here (Fig. 3).

\author{
Magomedov Rasul Magomedovich \\ $\mathrm{PhD}$ in Engineering sciences, Associate Professor \\ Dagestan State Institute of National Economy \\ Makhachkala, Russia \\ ventav@mail.ru
}

Akayev Abduldzhafar Imamuseynovich

$\mathrm{PhD}$ in Engineering sciences, Associate Professor

Dagestan State Institute of National Economy

Makhachkala, Russia

aabduldzhafar@mail.ru

\section{Chikayev Magomed Akhmedovich Senior lecturer \\ Dagestan State Institute of National Economy \\ Makhachkala, Russia \\ chikaev.magomed@mail.ru}

\section{PURPOSE OF STUDY}

The system of two nonlinear differential equations in partial derivatives of the mixed type, written relative to deflection function $\mathrm{W}$ and force function $\mathrm{F}$, describing the case of geometrically nonlinear deformation of depressed shells under the action of transverse load of intensity $q$ (dimensional values are marked by the wave) is taken as the basic one:

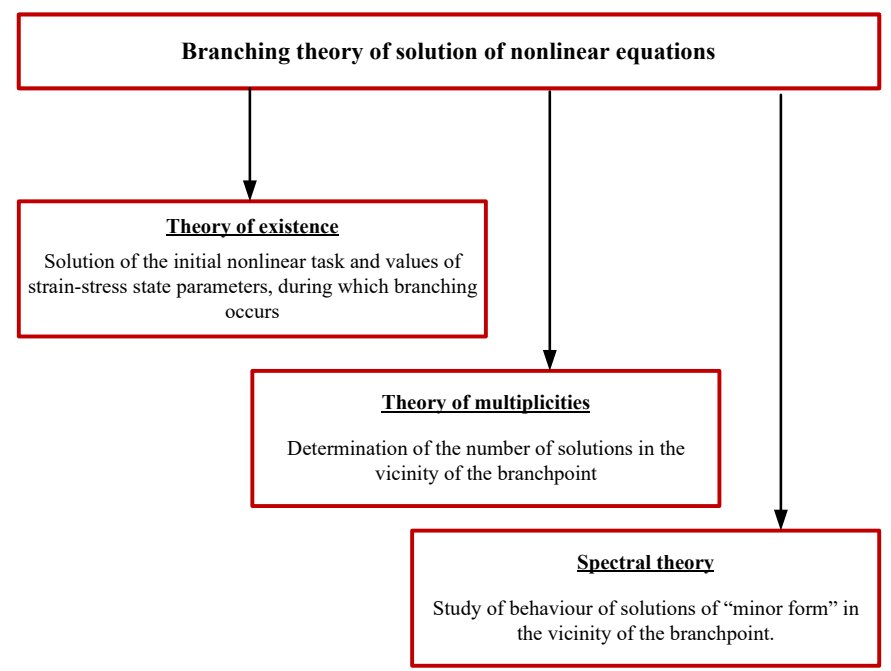

Fig. 1. Branching theory for solutions of nonlinear problems 


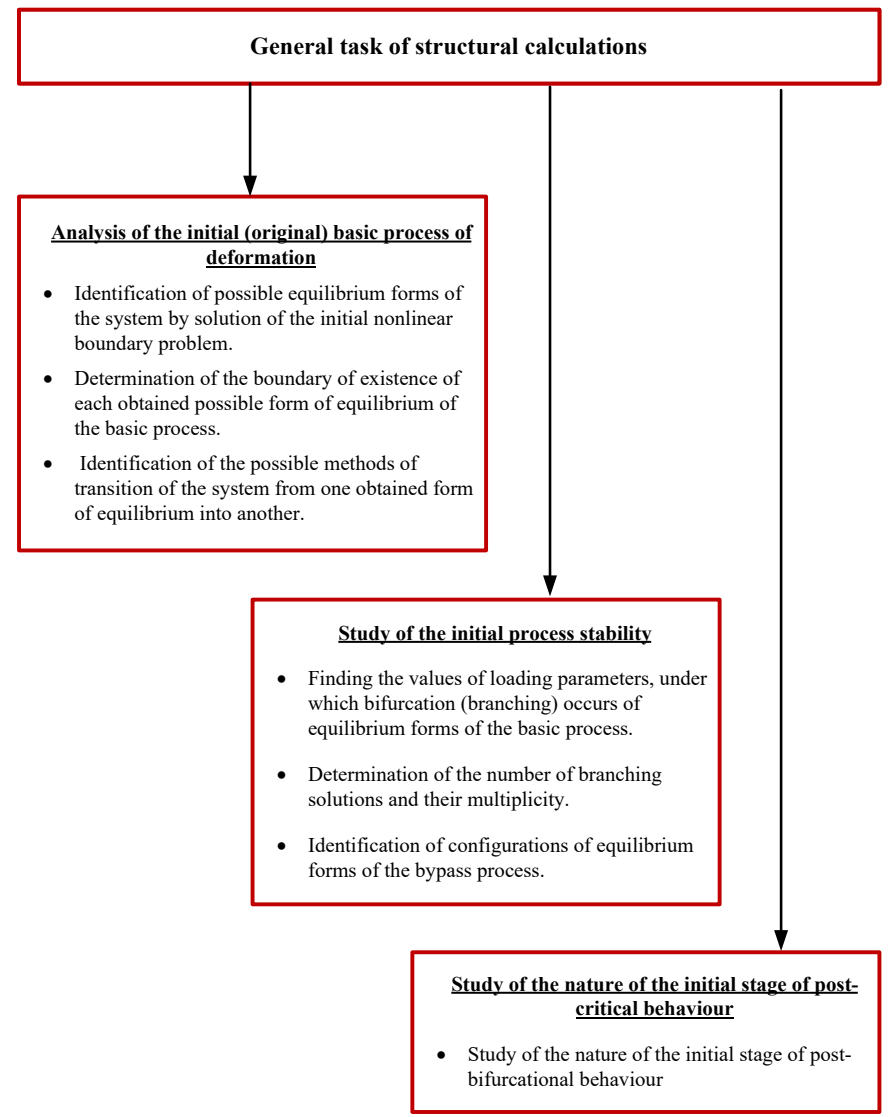

Fig. 2. General problem of structural analysis

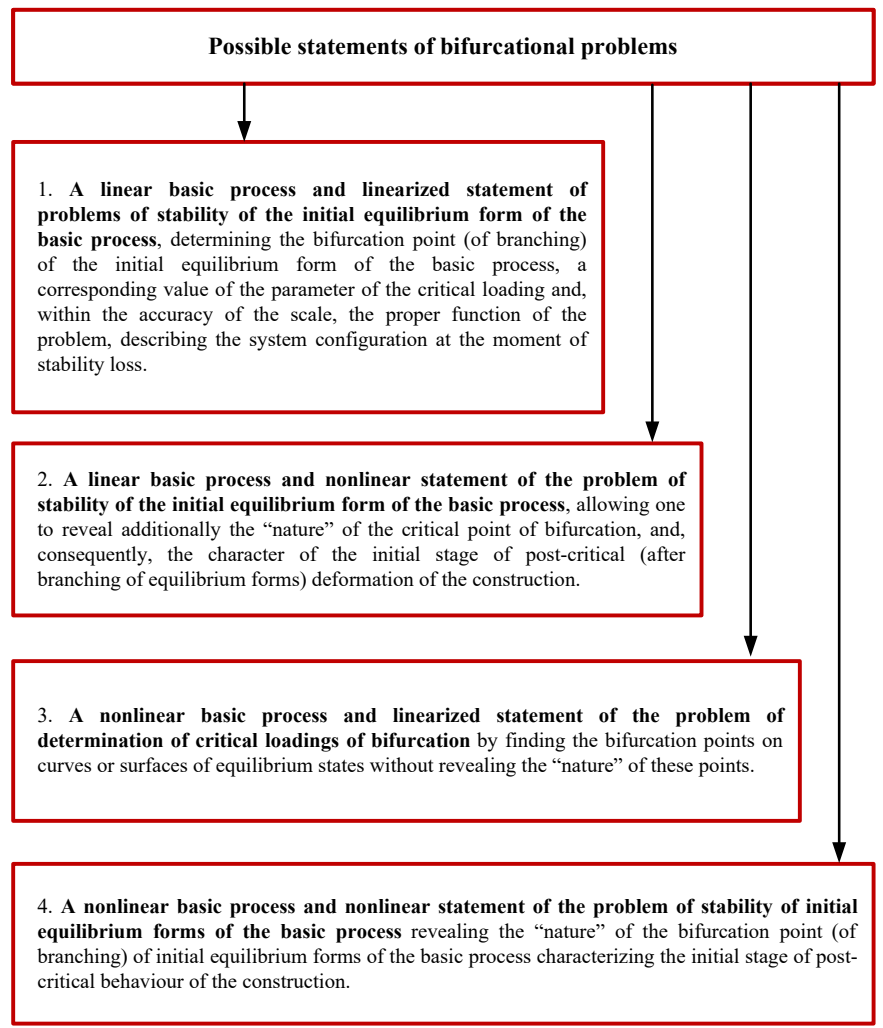

Fig. 3. Possible statements of bifurcation problems
In this work, because of an algorithm combining approximate analytical and numerical methods, one model problem is solved. It is the study of the peculiarities of behavior under load of a thin-walled spherical shell, by solving 4 types of problems from the ones shown in Fig.3.

The system of two nonlinear differential equations in partial derivatives of the mixed type, written relative to the deflection function $\mathrm{W}$ and the force function $\mathrm{F}$, describing a case of geometrically nonlinear deformation of shells under the action of a transverse load of intensity q (the wave size is observed) is taken as the main:

$$
\left\{\begin{array}{c}
D \cdot \nabla^{4} \widetilde{W}=\nabla_{\kappa}^{2} \widetilde{F}+L(W, F)+\widetilde{q} \\
(1 / E h) \cdot \nabla^{4} F=-\nabla_{\kappa}^{2} W-(1 / 2) \cdot L(W, W)
\end{array}\right.
$$

where $\nabla_{\mathrm{K}}^{2}(), \mathrm{L}()$ - differential operators having in polar coordinates the form:

$$
\begin{aligned}
\nabla_{\kappa}^{2}()= & K_{2} \frac{\partial^{2}()}{\partial r^{2}}+K_{1}\left[\frac{1}{r} \frac{\partial()}{\partial r}+\frac{1}{r^{2}} \frac{\partial^{2}()}{\partial \varphi^{2}}\right] \\
L(W, F)= & \frac{\partial^{2} W}{\partial r^{2}} \cdot\left(\frac{\partial F}{r \partial r}+\frac{1}{r^{2}} \frac{\partial^{2} F}{\partial \varphi^{2}}\right)+\frac{\partial^{2} F}{\partial r^{2}} \cdot\left(\frac{\partial W}{r \partial r}+\frac{1}{r^{2}} \frac{\partial^{2} W}{\partial \varphi^{2}}\right)+ \\
& -2 \frac{\partial}{\partial r}\left(\frac{\partial W}{r \partial \varphi}\right) \cdot \frac{\partial}{\partial r}\left(\frac{\partial F}{r \partial \varphi}\right)
\end{aligned}
$$

Replacing (3) F with W is the operator L (W, W).

The solution of these equations in a closed form, giving complete information about the features of the shell at all stages of loading is impossible. Therefore, in this article, the study of the behavior of the shell is divided into three stages, which can also be considered as separate tasks:

- nonlinear task of determining the stress-strain state at the initial - axisymmetric stage of work;

- determination of critical (bifurcation) load values;

- analysis of the nature of post-bifurcation behavior.

\section{METHODS}

1. In the case of axisymmetric deformation of the shell, the functions $\mathrm{W}$ and $\mathrm{F}$, and hence the components of the stressstrain state, do not depend on the polar angle $\varphi$. If, moreover, $K_{1}=K_{2}=K$, then the above formulas $(1) \div(3)$ are greatly simplified: system (1) can be integrated and its first integral takes the form:

$$
\left\{\begin{array}{c}
D \frac{d}{d r}\left(\nabla^{2} W\right)=h \frac{d F}{d r}\left(\frac{1}{r} \frac{d W}{d r}+K\right)+\Psi(r) \\
\frac{d}{d r}\left(\nabla^{2} \Phi\right)=-E\left[\frac{1}{2 r}\left(\frac{d W}{d r}\right)^{2}+K \frac{d W}{d r}\right],
\end{array}\right.
$$

where

$$
\nabla^{2}()=\frac{1}{r} \frac{d}{d r}\left[r \frac{d}{d r}()\right]-\text { Laplace operator in polar }
$$
coordinates for an axisymmetric problem; 
In the generally accepted dimensionless parameters and notation (Murtazaliyev G.M., 2004), the axisymmetric deformation equations (4) take the form:

$$
\left\{\begin{array}{c}
\left(r \cdot \Theta^{\prime}\right)^{\prime}-\frac{\Theta}{r}=\Phi(\Theta-r)-\Psi_{0}(r) ; \\
\left(r \cdot \Phi^{\prime}\right)^{\prime}-\frac{\Phi}{r}=-\Theta\left(\frac{\Theta}{2}-r\right),
\end{array}\right.
$$$$
\text { where } \Psi_{0}(r)=4 \int p r d r \text {. }
$$

In the case of loading the shell with a pressure of intensity $p$ applied to a circular region of radius $r_{1}$ centered at the top of the dome:

$$
\Psi_{0}(r)= \begin{cases}2 p r^{2}, & r<b_{1} ; \\ 2 p b^{2}, & r>b_{1},\end{cases}
$$

where $b_{1}=\frac{r_{1}}{a} b$.

In this case, the equations can be written in a uniform form:

$$
\left\{\begin{array}{c}
\left(r \cdot \Theta^{\prime}\right)^{\prime}-\frac{\Theta}{2}=\Phi(\Theta-r)-2 p \cdot b_{1}^{2} \cdot \delta(r) ; \\
\left(r \cdot \Phi^{\prime}\right)^{\prime}-\frac{\Phi}{r}=-\Theta\left(r+\frac{\Theta}{2}\right),
\end{array}\right.
$$$$
\text { where } \delta(r)= \begin{cases}\frac{r^{2}}{b_{1}^{2}}, & r<b_{1} ; \\ 1, & r>b_{1} .\end{cases}
$$

The system of equations (8) describes the elastic-nonlinear axisymmetric deformation of rotation shells; and together with the corresponding boundary conditions, they constitute a complete system of equations for solving problems.

This system can be solved by different methods. The main difficulty is to obtain a continuous curve of equilibrium states, since numerical methods become divergent in the vicinity of singular points. Previously, the authors of this work solved this problem based on an algorithm that combines the method of finite differences of increased accuracy with the method of differentiation by a parameter representing a "step-by-step" procedure. The procedure was used to change the leading parameter in the vicinity of singular points, which allowed one to construct continuous curves for different parameters of curvature and load, defining each point of each curve by solving a system of nonlinear equations.

The use of algebraic tools and geometric images of the theory of catastrophes is more effective. It allows presenting the behavior of entire classes of structures with a single clear geometric picture (Murtazaliyev G.M. 2004.). This procedure can significantly reduce subsequent calculations.

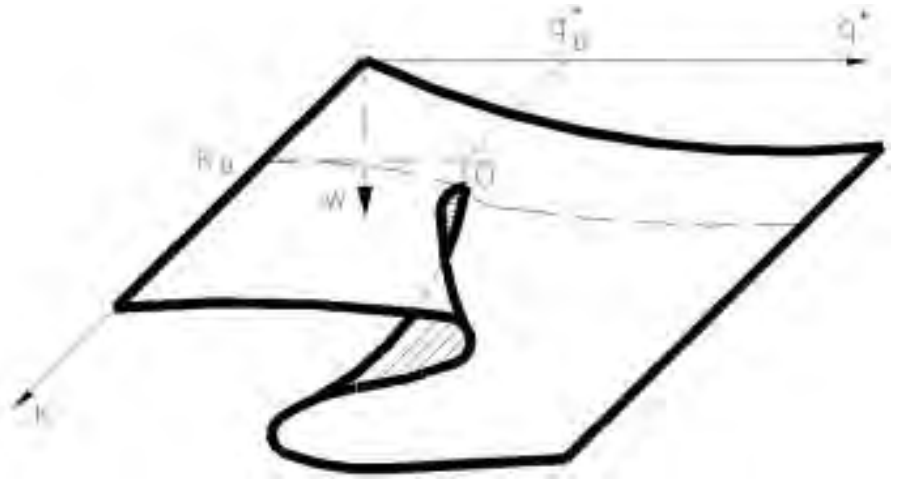

Fig. 4. Variety of assembly catastrophe. Surface of the equilibrium states of shell

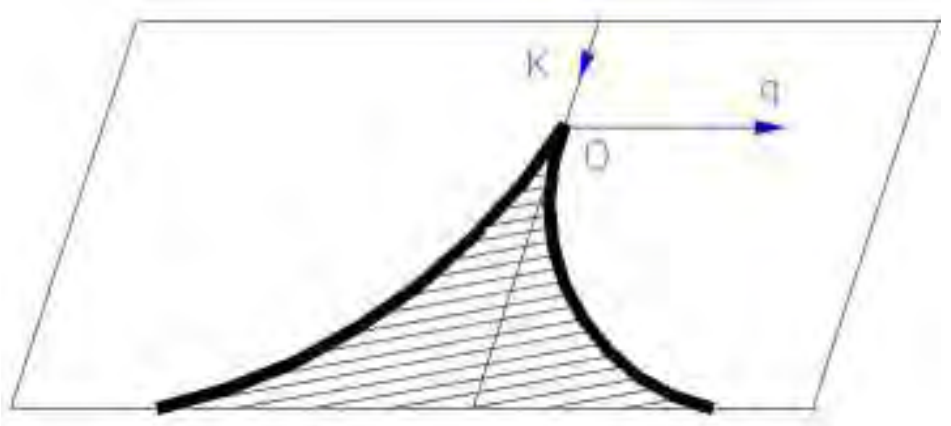

Fig. 5. Mapping of an assembly crash onto the plane of control parameters K and $\mathrm{q}$

In fig. 4 and 5, the manifold assembly catastrophe is shown: the surface of the equilibrium states of the shell and the mapping catastrophe of the assembly on the plane of the control parameters $\mathrm{K}$ and $\mathrm{q}^{*}$, giving complete qualitative and quantitative information about the behavior under load of an entire class of shells.

To calculate the critical loads of bifurcation, the function of deflection $\mathrm{W}$ and effort $\mathrm{F}$ at the moment of loss of stability in the form is presented:

$$
\begin{aligned}
& W(r, \varphi)=W_{0}(r)+\xi W_{1}(r, \varphi) ; \\
& F(r, \varphi)=F_{0}(r)+\xi F_{1}(r, \varphi),
\end{aligned}
$$

where Wo(r) и Fo(r) - values characterizing the axisymmetric behavior of shell;

$\mathrm{W}_{1}(\mathrm{r}, \varphi)$ and $\mathrm{F}_{1}(\mathrm{r}, \varphi)$ - increments of deflection functions and effort with a possible transition of the shell to adjacent asymmetrical forms;

\section{$\xi$ - infinitely law value.}

Substituting (10) into the original equations (1), considering equations (8) and the subsequent linearization with respect to $\xi$ leads to the equations: 


$$
\begin{aligned}
& \nabla^{4} W_{1}=\nabla^{2} F_{1}-\left(\frac{F_{1}}{r}-\frac{F_{1}}{r^{2}}\right) \Theta^{\prime}+\frac{\Phi}{r} W_{1}^{\prime \prime}-\frac{F_{1}^{\prime \prime}}{r} \Theta+\left(\frac{W_{1}}{r}+\frac{W_{1}}{r^{2}}\right) \Phi \\
& \nabla^{4} F_{1}=-\nabla^{2} W_{1}+\left(\frac{\dot{W}_{1}}{r}+\frac{\ddot{W}_{1}}{r^{2}}\right) \Theta^{\prime}+\frac{W_{1}^{\prime \prime}}{r} \Theta .
\end{aligned}
$$

These equations, besides the trivial solution $\left(\mathrm{W}_{1}=\mathrm{F}_{1}=0\right)$, for some values of the parameter of the external load included in the values of $\Theta$ and $\Phi$, have nontrivial solutions.

The functions $\mathrm{W}_{1}$ and $\mathrm{F}_{1}$ were taken in the following form:

$$
\begin{aligned}
& W_{1}(r, \varphi)=\sum_{n=2}^{\infty} W_{1 n}(r) \cos n \varphi ; \\
& F_{1}(r, \varphi)=\sum_{n=2}^{\infty} F_{1 n}(r) \cos n \varphi,
\end{aligned}
$$

where $\mathrm{n}-$ number of circumferential waves (the cases $n=0$ и $\mathrm{n}=1$ are special cases).

Considering (12), (11) turn into an infinite system of homogeneous ordinary differential equations for the functions $\mathrm{W}_{1 \mathrm{n}}(\mathrm{r})$ and $\mathrm{F}_{1 \mathrm{n}}(\mathrm{r})$ :

$$
\begin{aligned}
\nabla_{n}^{4} W_{1 n}= & \nabla_{n}^{2} F_{1 n}-\left(\frac{1}{r} F_{1 n}-\frac{n^{2}}{r^{2}} \ddot{F}_{1 n}\right) \Theta^{\prime}+\frac{\Phi}{r} W_{1 n}^{\prime \prime}- \\
& -\frac{\Theta}{r} F_{1 n}^{\prime \prime}+\left(\frac{1}{r} W_{1 n}-\frac{n^{2}}{r^{2}} W_{1 n}\right) \Phi^{\prime} ; \\
\nabla_{n}^{4} F_{1 n}= & -\nabla_{n}^{2} W_{1 n}+\frac{\Theta}{r} W_{1 n}^{\prime}+\left(\frac{1}{r} W_{1 n}-\frac{n^{2}}{r^{2}} W_{1 n}\right) \Theta^{\prime} .
\end{aligned}
$$

The system of equations by the method of finite differences of increased accuracy is reduced to a system of algebraic equations. The equality of the determinant to zero from the coefficients of the system of equations (13), in which the load parameter enters the values of $\Theta$ and $\Phi$ and determines the critical value of the load, at which the initial axially symmetric state turns into possible asymmetric forms.

3. To determine the nature of the initial stage of the postbifurcation state, we present:

$$
\begin{aligned}
& W=W_{0}+\xi W_{1}+\xi^{2} W_{2}+\ldots ; \\
& F=F_{0}+\xi F_{1}+\xi^{2} F_{2}+\ldots,
\end{aligned}
$$

where $\mathrm{W}_{0}$ and $\mathrm{F}_{0}$ - parameters of the original axisymmetric deformation;

$\mathrm{W}_{1}$ and $\mathrm{F}_{1}$ - normalized values of own functions characterizing the deflection;

$\mathrm{W}_{2}$ and $\mathrm{F}_{2}$ - functions characterizing the initial stage of the postcritical behavior of the shell to be determined; $\xi-$ a small parameter.

Substituting expressions (14) into the original equations (1), equating the coefficients with the same powers of the parameter $\xi$ in the right and left sides, considering equations (8) and (11), we obtain the following equations:

$$
\begin{aligned}
\nabla^{4} W_{2} & -\nabla^{2} F_{2}+\left(\frac{F_{2}^{\prime}}{r}+\frac{\ddot{F}_{2}}{r^{2}}\right) \cdot \Theta_{c r}^{\prime}+\frac{W_{2}^{\prime \prime}}{r} \Phi_{c r}+\frac{F_{2}^{\prime \prime}}{r} \Theta_{c r}-\left(\frac{W_{2}^{\prime}}{r}+\frac{\ddot{W}_{2}}{r^{2}}\right) \cdot \Phi_{c r}^{\prime}= \\
& =\left(\frac{F_{1}^{\prime}}{r}+\frac{\ddot{F}_{1}}{r^{2}}\right) \cdot \ddot{W}_{1}+\left(\frac{W_{1}^{\prime}}{r}+\frac{\ddot{W}_{1}}{r^{2}}\right) \cdot F_{1}^{\prime \prime}-2\left(\frac{\dot{F}_{1}}{r}\right)^{\prime}\left(\frac{\dot{W}_{1}}{r}\right)^{\prime} \\
\nabla^{4} F_{2}+ & \nabla^{2} W_{2}-\left(\frac{W_{2}^{\prime}}{r}+\frac{\ddot{W}_{2}}{r^{2}}\right) \cdot \Theta_{c r}^{\prime}-\frac{W_{2}^{\prime \prime}}{r} \cdot \Theta_{c r}= \\
& =\left(\frac{W_{1}^{\prime}}{r}-\frac{\ddot{W}_{1}}{r^{2}}\right)^{2}-\left(\frac{W_{1}^{\prime}}{r}+\frac{\ddot{W}_{1}}{r^{2}}\right) \cdot W_{1}^{\prime \prime}
\end{aligned}
$$

Equations (15) and the corresponding boundary conditions describe the post-bifurcation behavior of the shell in the initial part of the secondary equilibrium branch, i.e. in the vicinity of the branch point (bifurcation) of the original equilibrium form.

The analysis of the initial stage of post-bifurcation behavior is that of establishing the relationship between the parameter of the current load and the amplitude of the arising adjacent asymmetric shape of buckling. In this article, a variant of the general theory of stability and postcritical behavior of structures previously developed by V.T. Koyter. The algebraization of linear differential equations (15) is carried out by the finite differences method of increased accuracy with the subsequent solution of the resulting systems of equations using standard programs.

Because of such an algorithm, specific problems were solved, the values of the coefficients characterizing the initial stage of post-bifurcation behavior of the shells, and the relationships between the critical and limiting values of loads were obtained.

It is shown that depending on the area of the loaded part of the shell surface, the nature of the initial stage of postcritical deformation changes not only quantitatively but also qualitatively.

\section{CONCLUSION}

1. The proposed method for solving a general nonlinear boundary-value problem of structural analysis, consisting of three interconnected and successive stages, makes it possible to identify all the characteristic features of behavior under the load of thin-walled systems that lose their stability.

2. The most effective in solving problems associated with discontinuous phenomena are combinations of approximate analytical ones — catastrophe theories of catastrophes and numerical methods that do not require complicated, time-consuming, and significant computational volumes.

3. The analysis of the nature of the initial stage of postbifurcation behavior of structures allows one to assess the danger degree of reaching the critical state and, depending on the consequences, the guarantee against its occurrence must be different. It is achieved by considering the values of the corresponding reliability coefficients in the calculations. 


\section{References}

[1] V. I. Arnold. Catastrophe theory. Moscow, 2016.

[2] V. G. Bazhenov, E. G. Gonik, A. I. Kibets, D. V. Shoshin, Stability and limiting states of elastoplastic spherical shells under static and dynamic loads, Journal of Applied Mechanics and Technical Physics, vol. 1(55), 2014, pp. 13-22.

[3] A.I. Borodin, N.N. Novikova, N.N. Shash, "Use of synergistic methods and catastrophe theory", Effective crisis management, vol. 2(89), 2015, pp. 84-90.

[4] M. S. Ganeyeva, V. E. Moiseyeva, Nonlinear bending and stability of spherical and ellipsoidal shells under nonaxisymmetric loading, Strength of Materials, vol. 75, 2013,. pp. 105-114.

[5] K. S. Malykh, A. A. Novichkov, I. S. Pridatko, "Stability of spherical shells considering the initial irregularities of the form", In Proceedings of the 6th All-Russian Youth Scientific-Technical Conference. SaintPetersburg. pp. 62-64, 2014 [Youth. Technology. Space, 2014]

[6] G.M. Murtazaliyev, Methods of catastrophe theory in shell stability problems. Makhachkala, 2004.

[7] G.M. Murtazaliyev, A.M, Dibirgadzhiyev, M.A. Chikayev, "Methods of catastrophe theory in shell stability problems", Bulletin of the Dagestan State Technical University. Technical sciences, vol. 2 (44), 2017, pp. $162-172$.
[8] G.M. Murtazaliyev, M.M. Payzulayev, "Methods of catastroph theory in structural mechanics", November 27-28, 2015. Makhachkala: Tipografiya RIZO-PRESS, p. 132 [Theory of structures: achievements and problems: a collection of articles on the materials of the II AllRussian scientific-practical conference, 2015].

[9] G.M. Murtazaliyev, M.M. Payzulayev, S.V. Guseynova, "Geometric images of the catastrophe theory in nonlinear problems", Theory of structures: achievements and problems., November 19-20, 2012, Makhachkala. Makhachkala: Izd-vo DGTU. [Collection of articles based on the materials of the All-Russian scientific-practical conference, 2012, p. 126]

[10] V. A. Ostreykovskiy, Analysis of the dynamic systems' stability and controllability by the methods of the catastroph theory of: A manual for university students. Moskva: High School Publishing, 2005.

[11] V. V. Petrov, I. V. Krivoshein, "Influence of material inhomogeneity on the stability of nonlinearly deformable shallow shells of double curvature", Bulletin of the Saratov State Technical University. pp. 20-25, 2014.

[12] V. V. Pikul, "Shell Resistance", Problems of engineering and automation. 2. pp.81-87, 2012

[13] V. V. Semko, I. V. Krivoshein, "Modeling the influence of the type of boundary conditions on the stability of nonlinearly deformable shallow shells", Mathematical methods in engineering and technology, Nizhny Novgorod, pp. 53-55, 2013 [Proceedings of the 26th International Scientific Conference, 2013]. 